\title{
An adaptive dual-optimal path-planning technique for unmanned air vehicles
}

\author{
Clifford A. Whitfield* \\ The Ohio State University, 201 W. 19th Avenue, Columbus, Ohio 43210, USA
}

Received 15 November 2015 / Accepted 10 January 2016

\begin{abstract}
A multi-objective technique for unmanned air vehicle path-planning generation through task allocation has been developed. The dual-optimal path-planning technique generates real-time adaptive flight paths based on available flight windows and environmental influenced objectives. The environmentally-influenced flight condition determines the aircraft optimal orientation within a downstream virtual window of possible vehicle destinations that is based on the vehicle's kinematics. The intermittent results are then pursued by a dynamic optimization technique to determine the flight path. This path-planning technique is a multi-objective optimization procedure consisting of two goals that do not require additional information to combine the conflicting objectives into a single-objective. The technique was applied to solar-regenerative high altitude long endurance flight which can benefit significantly from an adaptive real-time path-planning technique. The objectives were to determine the minimum power required flight paths while maintaining maximum solar power for continual surveillance over an area of interest (AOI). The simulated path generation technique prolonged the flight duration over a sustained turn loiter flight path by approximately 2 months for a year of flight. The potential for prolonged solar powered flight was consistent for all latitude locations, including 2 months of available flight at $60^{\circ}$ latitude, where sustained turn flight was no longer capable.
\end{abstract}

Key words: Multi-objective optimization, Path-planning, Real-time, Unmanned air vehicle, Solar-powered flight.

\section{Introduction}

Unmanned Air Vehicle (UAV) classifications typically fall into one of six functional categories; however, recently unmanned systems have been developed for multi-role capabilities. The six functions include: target and decoy, reconnaissance, combat, logistics, civil and commercial, and research and development [1]. Regardless of the UAVs function, the basic essentials of unmanned vehicles include communication relay between the vehicle and ground operator for maintaining situational awareness, vehicle and payload sensors based on the UAV's function, and the use of global positioning systems to enable precise navigation. With the increase in radio frequency technology, the UAV became more sophisticated with the combined capability for remote and built-in control to perform low-level pilot duties such as flight-path stabilization. However, these vehicles were still not considered autonomous, commonly defined as the ability to make decisions without human intervention. UAV-autonomy technology falls under the following categories: sensor fusion, communications, path planning, trajectory generation (sometimes called motion

*e-mail: whitfield.22@osu.edu planning), trajectory regulation, task allocation and scheduling, and cooperative tactics [1]. A significant amount of effort has been focused in UAV autonomy, with the recent advances accredited to the field of control science. In fact, autonomy may continue to be the driving field for UAV development and expanding the UAV market.

Path-planning and trajectory generation and regulation methods for UAV flight have been investigated extensively, which include: evolution-based, extremum-seeking, randomized, neural-network, direct collocation with nonlinear programming, B-spline, quadtree data structuring, probabilistic approaches, Chebyshev pseudospectral, and fast graph search methods. Additionally, several algorithms have been developed, for example: evolutionary, random tree, Dijkstra, Reduced-State and Hierarchical Dijkstra, and A* search. Even investigations into the problem formulation of the dynamic and objective equations have been considered, such as an $n$th degree Lagrange polynomial approximation. Off-line and on-line approaches have been considered implementing, including others, the methods and algorithms listed; demonstrating the diversity of approaches in the path-planning category of UAV-autonomy [2-44]. To further categorize the path planning methods and trajectory generation efforts, consider 
the several goals and flight conditions that were investigated. Flight in a horizontal plane 2-D was analyzed [5, 6, 17, 33, 44], along with considering the effects of constant winds [2-4]. Employing obstacle avoidance [2, 27-32] and target tracking [33-41] within the trajectory generation was addressed. Path planning for multiple UAV formation and collision free cooperative control and tactical applications have been considered [42, 43]. Expanding beyond flight vehicles, even steady turns and optimal paths for underwater gliders have been investigated [45].

The underlying commonality of the work mentioned is the single-objective analysis. Typical single-objectives to pathplanning include time of flight or vehicle performance, such as endurance. Conflicting objectives when determining flight trajectories are generally unaccounted for. However, there are two main existing procedures that convert the multiple conflicting objectives into a single objective problem that have been used [2]. One method ( $\varepsilon$-constraint method), selects one of the objectives for the analysis while the others are used as constraints, restricting the objective within a safe limit. The other, the weighted-sum approach, combines all objectives to form a single-objective. Both procedures require artificial parameters, such as limiting values in the $\varepsilon$-constraint method or assigning relative weights in the weighted-sum procedure. Consequently, the results are dependent on the accuracy of the chosen parameters and, in the case of UAV flight tasks, choosing the parameters are not straight forward.

Concerns for the procedures should be addressed. For conversion of multiple objectives into a single objective, the objective types need to be consistent; either all of minimization or maximization type. With a conversion procedure using a weighted-vector, each objective must be normalized in magnitude prior to determining the weighted average. This requires relying on past experience or guessing when assigning the relative weight (or $\varepsilon$ ) vector.

New frameworks have been developed accounting for multiple objectives to UAV flight management. The operational objectives, generally, include: platform safety; minimizing fuel, time, distance; and minimizing deviation from the current path. The main commonalities of the recent developments have been the multiple solution paths generated based on predefined terrain criteria called meta-paths. The meta-paths, in excess of 15 or more, provide an off-line analysis and the decision, from the available generated paths, the "best" possible route. For instance; an off-line path-planning investigation using multiobjective evolutionary algorithms was considered by Mittal and Deb [2]. The procedure introduced a three-step hybrid algorithm. The steps of the algorithm first generated several path solutions, called Pareto-optimal, each with different trade-offs to the objective functions. With the generated solutions, 8-10 were selected providing trade-offs between the objectives; and locally searched to obtain a set of solutions close to the true Pareto-optimal front, of which a single flight path was to be selected. The off-line process required prior terrain topography information and representation in determining the UAV paths. Similarly, Foo et al. and Swarzentruber et al. $[46,47]$ used particle swarm optimization to generate multiple solutions based on predefined criteria. A single summarized representation of the 15 total alternate paths, for a particular preference was selected to form the meta-paths in the final decision making process.

The following introduces an adaptive multi-objective technique for UAV path and trajectory autonomy generation through task allocation. The technique is an adaptive path generation for UAVs considering multiple independent conflicting objectives without the necessity of developing artificial or weighting parameters. The technique generates a single flight path based on an environmentally-influenced objectively-driven flight window for the UAV. The process is locally-optimal based and adaptive, reducing, if not eliminating the "curse of dimensionality", which is often stated when describing the significant size in decision space required for off-line path search techniques. It is not limited by individual methods or algorithms, however, certain procedures in comparison are advantageous. The goal was to develop a technique that did not require off-line analysis of meta-paths to determine the "best" possible route, but rather a single adapting flight path furthering the UAV-autonomy. Due to the nature of the optimized condition, the path generated is locally constrained based on the influenced objectives; and may not present the "best" global optimum the off-line meta-paths provide. Additionally, the objectives were to verify and validate the technique to demonstrate its capability as an adaptive multi-objective real-time path-planning procedure, not to conduct an in-depth analysis of comparing and contrasting the many different algorithms available.

\section{Dual-optimal path-planning technique}

The Dual-Optimal Path-Planning (DOPP) technique utilizes dynamic optimization for determining optimal flight trajectories between continually updating optimal intermediate-states for the UAV based on environmentally-influenced objectives. The environmentally-influenced optimal condition, known as the "driver" determines the next condition, within a downstream virtual window of possible vehicle destinations and orientation built from the UAV kinematics. The step results are pursued by a dynamic optimization technique to determine an optimal flight path by minimizing a cost function subject to dynamic equation constraints, control inequality constraints, interior state equality/inequality constraints, and boundary conditions with initial and final states determined by the driveroptimized results.

This sequential technique is a multi-objective optimization procedure consisting of two goals without requiring additional information to combine the conflicting objectives into a singleobjective. The individual goals and objective complexity are not limited in type and are determined on an individual basis. The solving technique is also algorithm independent, allowing for versatility in the process of developing the vehicle trajectory. The technique expressed mathematically in general form is shown in Figure 1.

Notice the solution to the driver-objective determines the final time solution of $\mathbf{x}(t)$ for the path cost function. The path-objective analysis was based from classical optimal control theory and the powerful result known as the "minimum principle of Pontryagin". A visual of the basic DOPP flight 


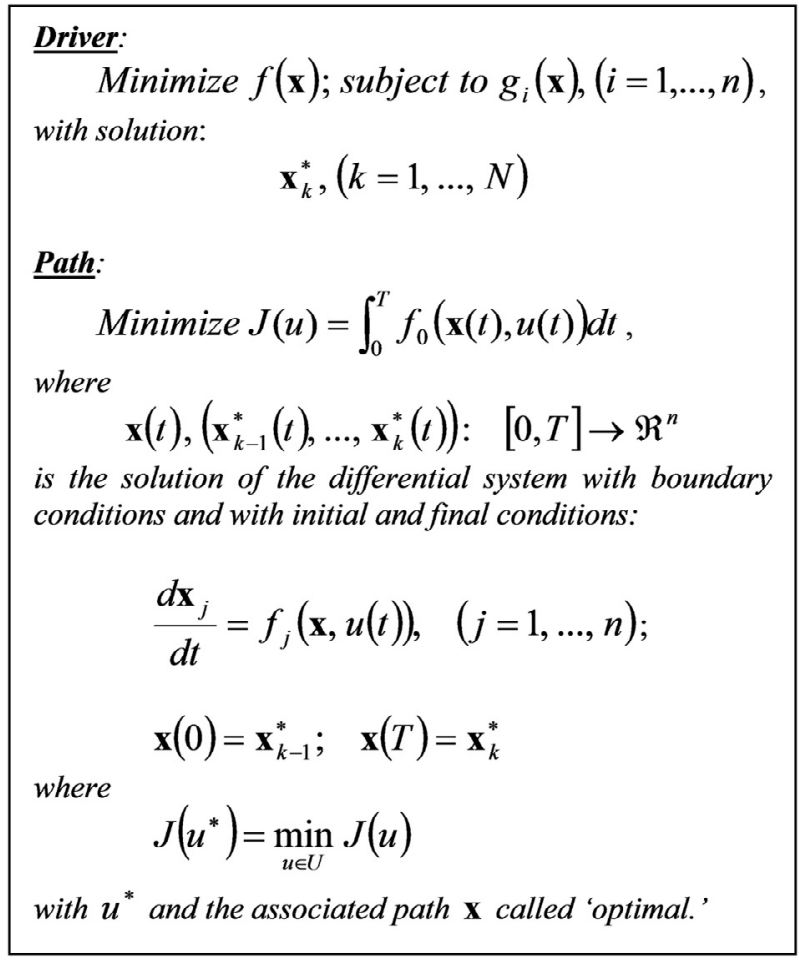

Figure 1. Dual-optimal path-planning technique.

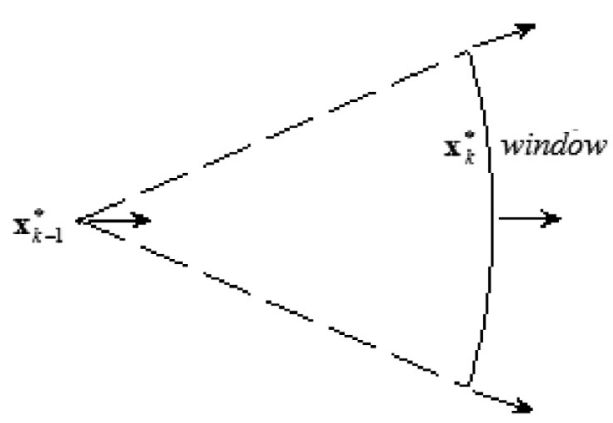

Figure 2. Dual-optimal path-planning planer flight maneuvering window.

window for the technique can be seen for planar flight in Figure 2.

The vehicles initial location $\mathbf{x}_{k-1}\left(\mathbf{x}_{k-1}^{*}\right.$ for an optimum) for planer flight consist of its location ( $x$ and $y$ ), velocity $(V)$, bank angle $(\phi)$, and heading $(\psi) ; f\left(\mathbf{x}_{k-1}\right)=f\left(x_{k-1}, y_{k-1}, V_{k-1}, \phi_{k-1}\right.$, $\left.\psi_{k-1}\right)$. In terms of three-dimensional, the vehicle's pitch angle and altitude are also required. At the initial location with the desired driver window projection distance, the available UAV flight window is projected forward of the heading direction and bounded based on the vehicle's kinematics.

For instance, looking at Figure 2, at the initial vehicle location $\mathbf{x}_{k-1}^{*}$, if the vehicle were to bank hard right (right wing down) would correspond to the farthest right location (with respect to initial heading) of the driver window. Similarly, a hard left bank would place the vehicle at the farthest left available position. Throughout the window, the vehicle has a $\mathbf{x}_{k}$ condition with corresponding location, velocity, bank and heading.
The environmentally-influenced driver-objective is optimized along this available flight window determining the $\mathbf{x}_{k}^{*}$ location and vehicle orientation. With that destination, the vehicle then proceeds from the initial to intermediate-state $\mathbf{x}_{k}^{*}$ location along an optimum flight path. The vehicle's path-objective, for example could be minimizing time, maximizing endurance, or minimizing power required. The process is then continued for all intermediate-states until the overall destination or time is reached. Three dimensional flight follows a similar setup that includes altitude change and UAV pitch flight characteristics.

Even though the technique is independent of algorithm, there are methods that are more suited for on-line processing. In general for the driver-objective search algorithms are advantageous, due to generating the available flight window from the UAVs flight mechanics. Regardless, in this technique the methods are required to be adaptive within the flight window (for all $N$ ), a case in point is an adaptive- $\mathrm{A}^{*}$ search algorithm.

A case study that could be benefit greatly from this technique was developed to verify and validate the technique. The case study focused on solar-regenerative extended flight (months) at high altitude for continual surveillance of an area of interest. For this example simulation, multiple objectives are predominate and developing off-line meta-paths are extremely, if not at all possible to take all variables into account through the entire flight duration. The technique was constrained using off-the-shelf technologies that are currently employed on UAVs, along with flight operations common for existing aircraft that provide high-altitude long-duration surveillance.

\section{Case-study}

High altitude flight above $60,000 \mathrm{ft}(18.3 \mathrm{~km})$, benefits from staying above turbulence, weather and air traffic, but comes with a price. As altitude increases the density decreases, requiring a larger lifting surface and a propulsion system that can operate in a low density atmosphere. These issues become more apparent for sustained on station persistent surveillance and reconnaissance flight. Continual surveillance over an area implies slow flight to maintain situational awareness. Slow flight in turn implies an increase in wing area and typically a decrease in wing loading, the ratio of the vehicle weight to the wings planform area. This encompasses its own challenges in vehicle design and operation. Regardless, there are current UAV platforms that reach these higher altitudes. The RQ-4 Global Hawk is one such UAV that has been used for reconnaissance, but it has a limited fuel capacity which results in a limited endurance and thus, restricted surveillance capability. Alternate methods for propulsion systems to potentially replace conventional fuel systems and increase endurance capability have been investigated. One method utilizes the photovoltaic solar panel to gather power from the Sun, and has been adapted for use on high altitude UAV platforms. An aircraft that uses solar panels and powered by the Sun during the day and stored energy through the night by solar regenerative power systems, in principle could sustain flight for weeks or months. Only mechanical wear would limit the flight duration.

The case-study application for long duration flight demonstrates the potential of the DOPP technique, and considers the 
change in ephemeris effects throughout the year. The casestudy is limited to a constant altitude of $60,000 \mathrm{ft}(18.3 \mathrm{~km})$, and the flight window is set based on existing surveillance package capabilities to restrict the vehicle's flight paths to provide and maintain continual situational awareness over an area of interest. A summary of the requirements and limitations for a solar-regenerative high altitude long endurance UAV is discussed. A more in-depth analysis is provided in reference [48].

\subsection{Requirements and limitations}

There are three main factors that need to be considered to appropriately model the DOPP technique: the environment (ephemeris effects, available solar flux at altitude, and highaltitude winds at a given location), the UAV systems (photovoltaics, energy storage system, electric motor requirements, and surveillance equipment limitations at altitude), and the UAV platform (vehicle geometry and flight mechanics).

\subsubsection{Flight environment}

The maximum amount of available solar power depends on the vehicle's geometry and flight orientation relative to the Sun's instantaneous location. The orientation is determined by the surface's obliquity factor, $\cos (\theta)$ and is determined by the local solar coordinates $(Z$ and $A)$ and the tilt coordinates of the surface ( $\Delta$ and $\Psi$ ). The obliquity factor is given by:

$$
\cos \theta=\cos Z \cos \Delta+\sin Z \sin \Delta \cos (A-\Psi)
$$

The first tilt coordinate $(\Delta)$ is the angle between surface normal and the vertical. The second $(\Psi)$ is the angle between the projection of the surface normal in the horizontal plane and due south direction. The tilt coordinates fix the direction of the surface normal. This is similar in the same manner as the solar coordinates in fixing the direction of the Sun's rays.

The model used for tracing the Sun through the sky at any given location and duration of time was the described by Weider [49], and determines the local solar coordinates and the zenith angle ( $\mathrm{Z}$ ) and azimuth angle (A) for an observer through the geocentric coordinates. The geocentric coordinate system has an origin at the center of the Earth with the $z$-axis connecting the north-south poles. The other two axes lie on the equatorial plane with the $x$-axis oriented toward the local (chosen) meridian.

For UAV flight operations over a long period (months), the equation of time was implemented within the model to account for the cumulative effect of the slight variations in the solar day throughout the entire year. The variation in time can increase or decrease the total solar day by as much as 16 min, dependent on the time of year, and cannot be ignored for long duration solar powered flight.

Within Earth's atmosphere, the solar intensity felt on a surface is composed of the direct and diffuse flux components ( $F^{\text {(direct) }}$ and $F^{\text {(diffuse) }}$, respectively). The latter term is the radiation that is scattered due to the atmosphere and the reflected radiation due to an underlying terrain. For the DOPP technique application, three assumptions will be made in order to develop a semiquantitative analysis of solar flux: (1) the diffuse flux due to the scattering of the solar intensity through the atmosphere has a significantly smaller effect than the direct flux component. This assumption is often used for approximating solar intensity at the Earth's surface. The validity of this assumption increases at high altitudes with the solar radiation traveling through less of the atmosphere, (2) the downward diffuse flux on the surface due to scattering radiation is isotropic, and (3) the upward diffuse flux on the surface will be considered negligible and disregarded in the formulation of the analysis. The upward diffuse flux is on the order of the reflective radiation component due to the underlying Earth's surface. With increase in the altitude the Earth's reflectivity radiation decreases, and for high altitude applications, the effects of the upward diffuse flux will be comparatively less to the downward diffuse flux component. With these assumptions the following equation will be used in determine the total solar flux:

$$
F=F^{(\text {direct })}+\left[\frac{1+\cos \Delta}{2}\right] F^{\downarrow \text { (diffuse })}
$$

where,

$$
F^{\text {(direct) }}=S_{0} \mu e^{-\tau / \mu_{0}}
$$

and,

$$
F^{\downarrow(\text { diffuse })}=S_{0} \mu_{0}\left[\frac{1}{1+G}\left(G e^{\gamma^{+} \tau}+e^{\gamma^{-} \tau}\right)-e^{-\tau / \mu_{0}}\right]
$$

$S_{0}$ is the solar constant $\left(125.6 \mathrm{~W} / \mathrm{ft}^{2}\right.$ or $\left.1352 \mathrm{~W} / \mathrm{m}^{2}\right)$. The surface orientation parameters are the tilt angle $\cos (\Delta)$, obliquity of the panel to the Sun's rays $\cos (\theta)$, and the cosine of the solar zenith angle $\mu_{0}=\cos (Z)$. The equations include two environmental parameters, the optical thickness $(\tau)$ and the single scattering albedo $\left(\tilde{\omega}_{0}\right)$ within the variables $G$, and $\gamma^{ \pm}$:

$$
\begin{gathered}
G=-\left[\frac{\gamma^{-}+A}{\gamma^{+}+A}\right] e^{\left(\gamma^{-}-\gamma^{+}\right) \tau} \\
\gamma^{ \pm}=1 / 2(C-A) \pm 1 / 2\left[(C-A)^{2}-4 B D\right]^{1 / 2}
\end{gathered}
$$

where,

$$
\begin{array}{rlrl}
A & =\frac{2-\tilde{\omega}_{0}}{2 \mu_{0}} & B & =\tilde{\omega}_{0} \\
C & =2-\tilde{\omega}_{0} & D & =\frac{\tilde{\omega}_{0}}{2 \mu_{0}}
\end{array}
$$

The environmental parameters are functions of the Sun's ray wavelengths. For the parameters, Rayleigh's law is commonly used in which the theory suggests that the scattering of solar energy varies smoothly with wavelength. This law is based on the altitude conditions, mean molecular mass of air, the index of refraction of the atmosphere and the constant Avogadro's number. For a relatively clear atmosphere the averaged optical thickness and the scattering is less than 0.3 and 0.6 respectively. Considering a completely horizontal surface $\left(\Delta=\mu=Z=0^{\circ}\right)$, the solar flux increases with altitude from $93 \mathrm{~W} / \mathrm{ft}^{2}\left(1000 \mathrm{~W} / \mathrm{m}^{2}\right.$ or $\left.1 \mathrm{Sun}\right)$ at the Earth's surface to slightly over $121 \mathrm{~W} / \mathrm{ft}^{2}\left(1300 \mathrm{~W} / \mathrm{m}^{2}\right.$ or $\left.1.3 \mathrm{Sun}\right)$ at 


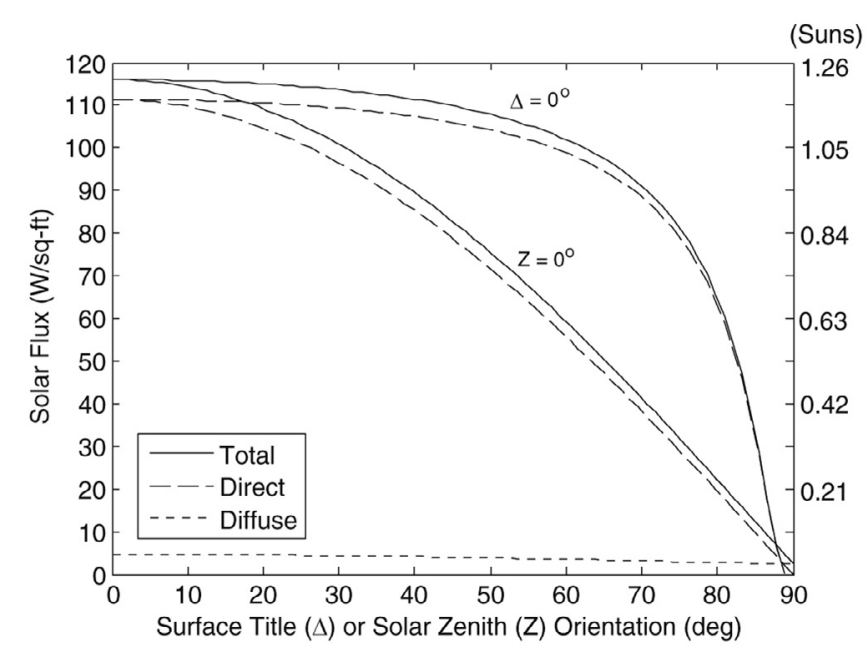

Figure 3. Solar flux with surface orientation at 60,000 ft (18.3 km) altitude.

$82,000 \mathrm{ft}(25 \mathrm{~km})$ altitude. Also, note that in terms of orientation the component for the solar intensity due to the downward diffuse flux is only a function of the cosine of the solar zenith and independent of the tilt and obliquity of the surface.

For a solar powered UAV at an altitude of $60,000 \mathrm{ft}$ $(18.3 \mathrm{~km})$ and a surface oriented horizontally, the maximum solar flux is $116.1 \mathrm{~W} / \mathrm{ft}^{2}\left(1250 \mathrm{~W} / \mathrm{m}^{2}\right.$ or $\left.1.25 \mathrm{Sun}\right)$. Of which, the direct solar flux is $111.5 \mathrm{~W} / \mathrm{ft}^{2}\left(1200 \mathrm{~W} / \mathrm{m}^{2}\right.$ or $\left.1.2 \mathrm{Sun}\right)$ and the downward diffuse flux is $4.6 \mathrm{~W} / \mathrm{ft}^{2}\left(50 \mathrm{~W} / \mathrm{m}^{2}\right.$ or 0.0046 Sun), $4 \%$ of the total. The total, direct and diffuse solar flux as a function of orientation for the case-study altitude of $60,000 \mathrm{ft}(18.3 \mathrm{~km})$ is shown in Figure 3.

The two conditions in Figure 3 are variation in surface title angle $\Delta$ with Sun in a constant location, zero solar zenith angle $\left(Z=0^{\circ}\right)$, and the variation in solar zenith angle from sunrise/ set to solar noon with the surface normal to the Sun for all zenith angles, zero surface title angle $\left(\Delta=0^{\circ}\right)$.

In addition to the available solar flux at high altitudes, and the based on the fact that high-altitude vehicles of this nature have significantly low wing-loading characteristics, the pervasive winds at those altitudes must also be considered. A study of the upper winds (ranging from 100 to $25 \mathrm{mb}$ pressure altitudes) for high altitude platform design was conducted in excess of a 20 year span over four areas of interest [50]. These areas include the contiguous United States, the Norwegian area, the Mediterranean area and the Pacifica area from Alaska to Japan and the surrounding regions. Results of the investigations shown that high altitude wind speeds were at a minimum for altitude ranges of 59,000-72,000 ft (18-22 km). Table 1 summarizes the average high altitude wind speed for each of the United States, case study location.

Wind roses are provided as an appendix of reference [50] for every $10 \mathrm{mb}$ pressure altitude throughout the four locations and seasons.

\subsubsection{UAV systems}

The UAV systems, common to high altitude surveillance aircraft, are discussed in order to set constraints for the fixed
Table 1. Average wind speeds.

\begin{tabular}{lc}
\hline Season & United States \\
\hline Winter & $30 \mathrm{kn}$ \\
Spring & $15 \mathrm{kn}$ \\
Summer & $25 \mathrm{kn}$ \\
Fall & $15 \mathrm{kn}$ \\
\hline
\end{tabular}

and optimized flight pattern analysis. The systems were chosen based on existing equipment that have been proven and used in both solar-regenerative and surveillance platforms; these include: photovoltaics, energy storage system, electric motors, and surveillance equipment.

A semiquantitative empirical analysis approach [49] was taken to model the photovoltaic expected available power equation (8) and photovoltaic efficiency equation (9):

$$
\begin{gathered}
P_{A}=V J \\
\eta=\frac{P_{A}}{F}=\frac{V J}{F}
\end{gathered}
$$

where, the current $(J)$ supplied to an external load is a combination of the photocurrent $\left(J_{\mathrm{p}}\right)$ through radiative energy or flux and the junction current $\left(J_{\mathrm{j}}\right)$ shunted by the diodes of the photovoltaic material:

$$
J=J_{\mathrm{p}}-J_{\mathrm{j}}
$$

Applying the modeling approach was done in conjunction to matching overall maximum power available from a representative UAV, NASA Pathfinder. This representative UAV was chosen due to its demonstrated flight tests and promising potential for high-altitude solar-powered long endurance flight.

At $60,000 \mathrm{ft}(18.3 \mathrm{~km})$ and at solar noon the maximum solar flux is $111.5 \mathrm{~W} / \mathrm{ft}^{2}\left(1200 \mathrm{~W} / \mathrm{m}^{2}\right.$ or $\left.1.2 \mathrm{Sun}\right)$. With $75 \%$ of the upper wing surface covered in solar panels, the amount of power capable of being generated at solar noon was $8000 \mathrm{~W}$. Physical parameters for the empirical analysis were adjusted within appropriate bounds to match recorded power available at altitude during solar noon. With the expected photovoltaic parameters modeled, Figure 4 shows the cell array solar energy characteristics at $60,000 \mathrm{ft}(18.3 \mathrm{~km})$.

Energy storage systems are key in sustaining continuous surveillance capability for solar-regenerative high altitude UAVs. This becomes particularly apparent with the decreasing daylight hours during the winter months for the northern hemisphere, and the energy per mass capacity $\mathrm{W} \mathrm{hr} / \mathrm{lbs}(\mathrm{W} \mathrm{hr} / \mathrm{kg})$ that can be stored during the daylight is critical. Lithium-sulfur batteries, from Sion Power have reported energy/mass values of $778.1 \mathrm{~W} \mathrm{hr} / \mathrm{lbs}(350 \mathrm{~W} \mathrm{hr} / \mathrm{kg})$; while Lynntech's Gen5 fuel cell technology [51] has shown experimental performance of $1411 \mathrm{~W} \mathrm{hr} / \mathrm{lbs}(640 \mathrm{~W} \mathrm{hr} / \mathrm{kg})$. For the case-study UAV platform, a realistic representative weight from existing aircraft for the energy storage system was selected and used throughout the example discussion for fixed and optimized flight patterns.

Lightweight brushless direct current electric motors and their performance were selected based on existing technology 

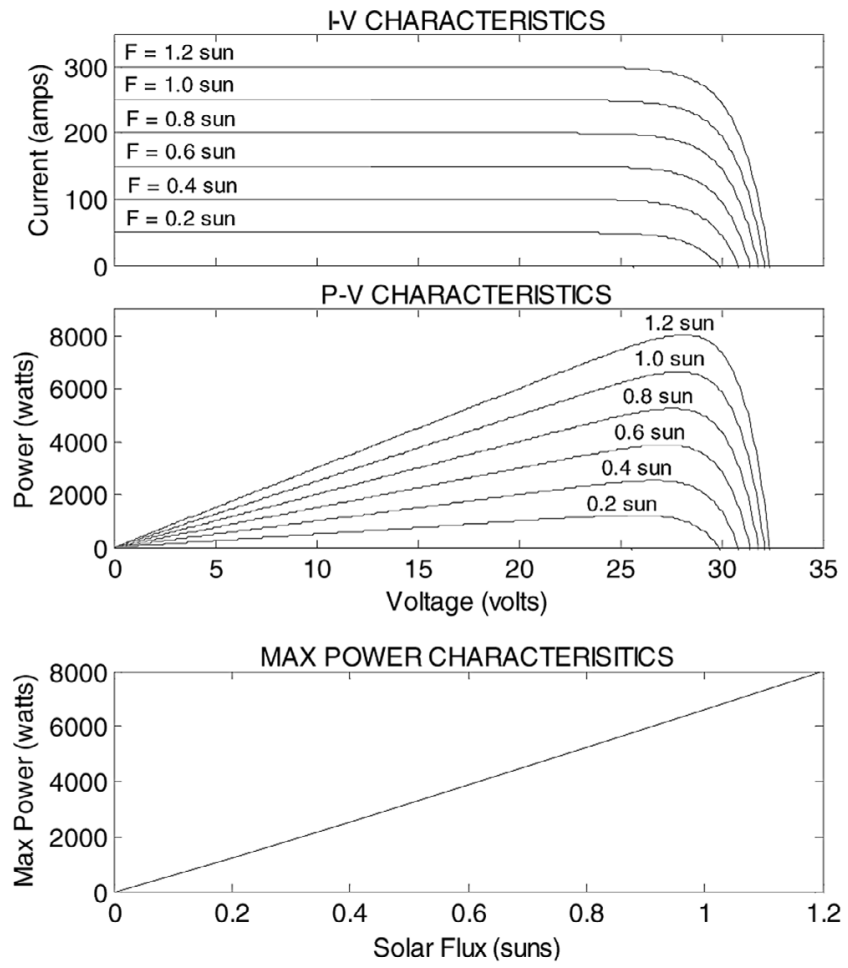

Figure 4. Solar Cell Array Characteristic at 60,000 ft (18.3 km).

that has been incorporated and tested on high-altitude UAV platforms [48]. The gearless motors are capable of producing 1.5 horsepower $(1.25 \mathrm{~kW})$ each.

The specifications of the surveillance equipment were used in determining the flight window constraints for the high altitude UAV operation. For instance, for a vehicle flying at an altitude of $60,000 \mathrm{ft}(18.3 \mathrm{~km})$ would have a line-of-sight that correlates to $90.260^{\circ}$ (sunrise/set) in longitude, or a distance of up to 6220 mile $(10,010.1 \mathrm{~km})$ radius (at the equator). However, to process any detail on the Earth's surface from that altitude the surveillance equipment determines the maximum "range-of-sight" to maintain situational awareness of an AOI on the Earth's surface for the vehicle.

For the case-study analysis and overall program capability demonstration, the Hughes Integrated Surveillance \& Reconnaissance sensor system was used [51]. This system has been carried on the Global Hawk, a UAV performing ISR missions with an on station endurance of 24-30 h, and integrates three separate systems controlled through one processor for expanding surveillance capabilities. The operational modes are; (1) wide-area that can detect movement within a radius of 62 miles $(99.8 \mathrm{~km})$, (2) strip-mode that provides $20 \mathrm{ft}$ $(6.1 \mathrm{~m})$ resolution over a 23 mile $(37 \mathrm{~km})$ wide region, and (3) spot-mode that can provide $6 \mathrm{ft}(1.8 \mathrm{~m})$ of resolution over a 3.8 mile $(6.1 \mathrm{~km})$ region with sea-surveillance capability.

The solar-powered flight pattern analysis approach taken was to demonstrate optimal performance for the vehicle in range-of-sight for wide-area mode, and provided a boundary constraint for the optimal flight paths generated real-time by the DOPP technique at altitude. If a target was acquired, and more detailed surveillance required, then a pattern-transfer
Table 2. Representative UAV platform summary [48, 52].

\begin{tabular}{ll}
\hline Platform & \multicolumn{1}{c}{ Performance } \\
\hline Weight: $550 \mathrm{lbs}$ & Altitude: $60,000 \mathrm{ft}$ \\
Wing loading: $0.7 \mathrm{lbs} / \mathrm{ft}^{2}$ & Pressure: $151 \mathrm{lbs} / \mathrm{ft}^{2}$ \\
Power loading: $13.5 \mathrm{~W} / \mathrm{ft}^{2}$ & Temperature: $-69.7{ }^{\circ} \mathrm{F}$ \\
Geometry & \\
Span: $98.5 \mathrm{ft}$ & Profile drag coefficient: 0.0170 \\
Length: $12.0 \mathrm{ft}$ & Max lift coefficient: 1.3 \\
Chord: $8.0 \mathrm{ft}$ & Efficiency factor: 0.92 \\
Aspect ratio: 2.0 & Max lift-to-drag ratio: 22.6 \\
\hline
\end{tabular}

would be taken to a new smaller range-of-sight window constraint for the strip-mode or the spot-mode function.

\subsubsection{UAV platform}

Similar geometric characteristics and flight mechanics of a representative UAV was selected for the case-study based on its promising flight tests [52]. The representative UAV is a flying wing with a high aspect ratio, resulting in very low wig loadings, less than one pound per square foot, limiting the onstation flight capability. Table 2 summarizes the representative UAV platform and aerodynamic performance characteristics.

With the wind tunnel and flight test data that has been published [52], this solar powered vehicle demonstrated the capability for sustained flight at high altitude.

\subsection{Baseline flight pattern}

The sustained turn flight, or loiter pattern, is a logical choice for maintaining continual situational awareness. This flight pattern was selected as the baseline for DOPP technique comparisons. The loitered flight has several dependent flight variables, such as the turn radius is set by the UAVs speed and bank angle. Therefore, consider the representative UAV platform required power in order to maintain a sustained turn at various bank angles shown in Figure 5. The maximum available power and the vehicle's aerodynamic stall velocity are included, showing the feasible velocity range for turning flight.

Referring to Figure 5, the minimum power required for sustained bank angles occurs at the vehicle's stall. Allowing for a $20 \%$ safety factor in vehicle speed above stall, the velocity for minimum power required for the representative UAV was set at a constant $50 \mathrm{kn}$. At this velocity the maximum aerodynamic efficiency for the vehicle was maintained at a lift coefficient of 0.85 and lift-to-drag ratio of 22 for level flight. The velocity setting also allows bank angles for the UAV up to $\pm 20^{\circ}$ without getting dangerously close to losing the necessary lift to maintain flight. With further increase in bank, the structural integrity along with the potential for the vehicle to stall would be of concern, especially with the vehicle's low wing loading.

At $50 \mathrm{kn}$ the vehicle requires an angle-of-attack of $10^{\circ}$. While the bank angle is increased to its maximum limit of $\pm 20^{\circ}$, the required change in lift and therefore change in angle-of-attack for constant speed is less than half of degree. This minimal change was ignored for the analysis; and the angle-of-attack was assumed a constant $10^{\circ}$ and accounted 


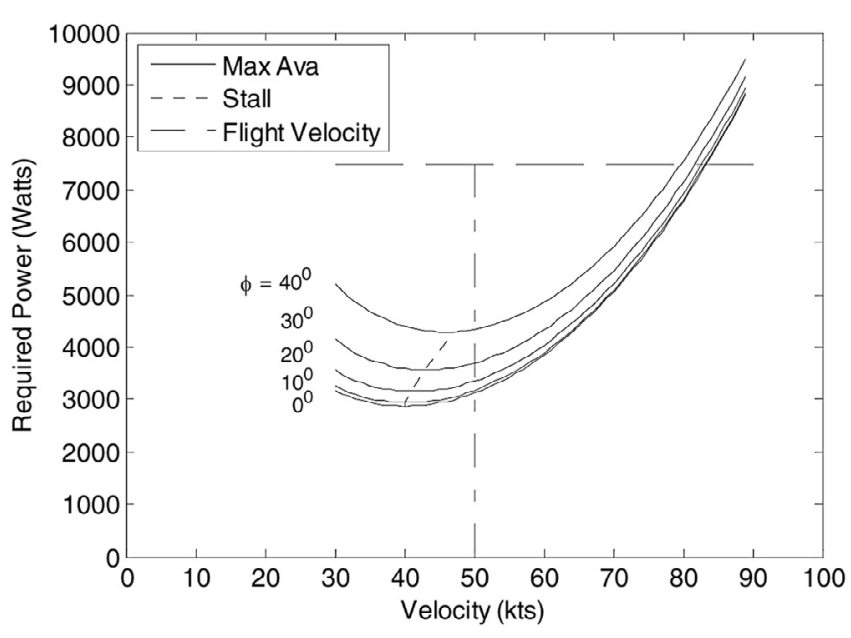

Figure 5. Sustained turn required power.

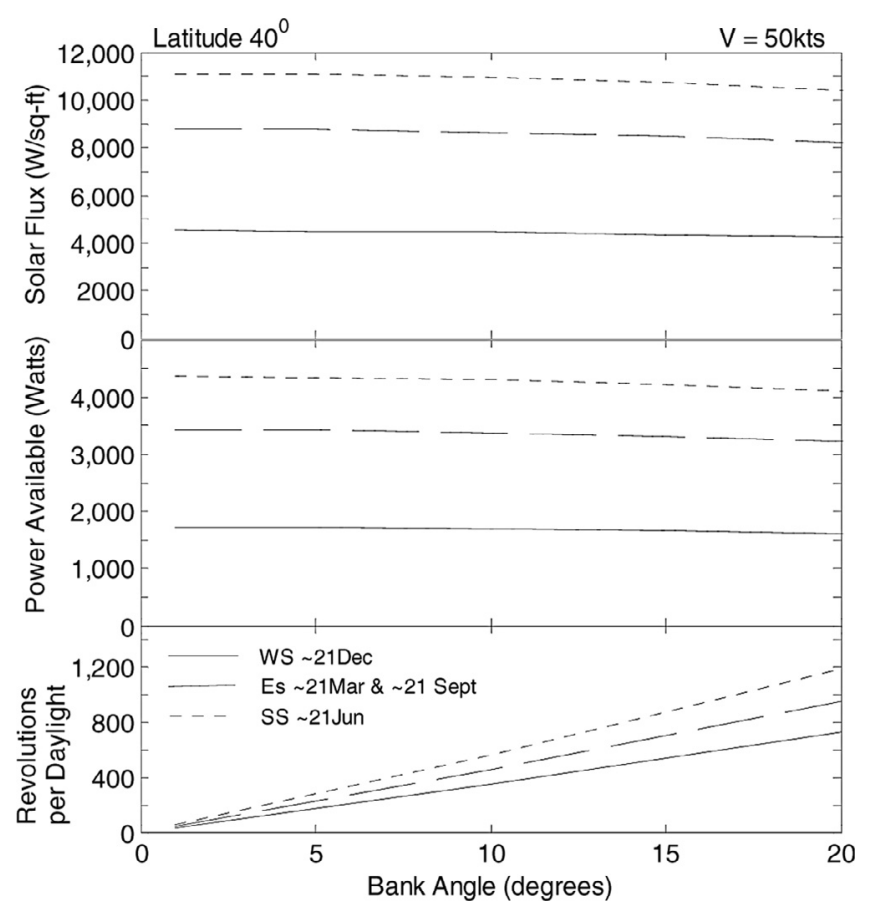

Figure 6. Total sustained turn solar flux and available power.

for in the surface obliquity tilt angles for determining the solar flux. Figure 6 shows the sustained turn analysis for the UAV for varying the bank angle at latitude of $40^{\circ}$.

The above figure shows the total solar flux and corresponding averaged power available for the winter and summer solstices and equinoxes, as well as the revolutions per daylight (RPDL) for constant speed flight. Comparing the averaged available power of Figure 5 to the power required for flight in Figure 6, it can be seen that the representative UAV does not gather enough solar power during the winter solstice to maintain flight, and also limited in available flight duration during the equinox times-of-year.

The other parameters of interest when considering sustained turn flight is the initial starting location at sunrise and the direction, clockwise $\left(D_{\mathrm{C}}\right)$ or counterclockwise $\left(D_{\mathrm{C}-\mathrm{C}}\right)$.
However when considering these parameter and due to the fact that the UAV has high revolutions per daylight the effects are inconsequential. This can be seen in Table 3 .

The vehicle conditions were sustained $\pm 20^{\circ}$ bank angle at a constant $50 \mathrm{kn}$ over the case-study location. The initial vehicle locations at sunrise were the four main cardinal directions; North, South, East, and West. With a turning radius of 0.10 nautical-miles $(608 \mathrm{ft})$, notice the high revolutions per day light and range over the time-of-year for the UAV. This is evident by observing the solar flux variation from sunrise to sunset for the vehicle in a sustained turn. Figure 7 shows the solar flux variation for the solstices and equinoxes.

The revolutions per daylight are shown in the figure for each time-of-year. The total solar fluxes listed in Table 3 are the integrand of solar flux variation of the above figure. Changing the initial cardinal direction at sunrise the solar flux frequency and peak magnitudes are maintained, with different sunrise conditions.

Considering the changes in latitude, the total solar flux and averaged available power, along with the revolutions per daylight for the UAV can be seen in Figure 8.

The flight conditions are consistent with the previous analysis, $20^{\circ}$ bank angle at a constant $50 \mathrm{kn}$. Recall that the power required for the vehicle, from Figure 5 is $3350 \mathrm{~W}$ for the bank angle. The requirement limits the possible sustained turn, loiter flight for the representative UAV in maintaining situational awareness; especially during the winter solstice, where there is only sufficient power for latitudes ranging from the equator to approximately $15^{\circ}$.

\subsection{DOPP case study modeling}

The UAV kinematic modeling considered is shown below equation (11). The vehicle is at a constant altitude, speed $(V)$ and angle-of-attack.

$$
[\mathbf{x}]=\left[\begin{array}{c}
x \\
y \\
\psi
\end{array}\right] \quad[\dot{\mathbf{x}}]=\left[\begin{array}{c}
V \cos \psi+V_{W x} \\
V \sin \psi+V_{W y} \\
(g V) \tan \phi
\end{array}\right]
$$

The position and orientation of the vehicle is given by the Cartesian coordinates ( $x$ and $y$ ), vehicle heading $(\psi)$ and bank angle $(\phi)$.

By introducing the bang-level-bang technique for vehicle control, the dependence on inertial roll rate and its effect to surface obliquity is eliminated in the near time control for vehicle orientation to meet its "driven" determined position and orientation. This simplification assumes that the vehicle has the ability to instantaneous reach a new bank angle and is common in flight vehicle trajectory analysis. Furthermore, the nature of the UAV's slow flight does not require large instantaneous changes in vehicle bank angle, eliminating the need for large roll rates, further validating the use of the UAV's control technique.

Recall that the objective was to determine the minimum required power flight paths to the predetermined location and orientation for obtaining maximum solar flux established by the "driver". Along the available window of flight of longitude 
Table 3. Total sustained turn solar flux with initial start location.

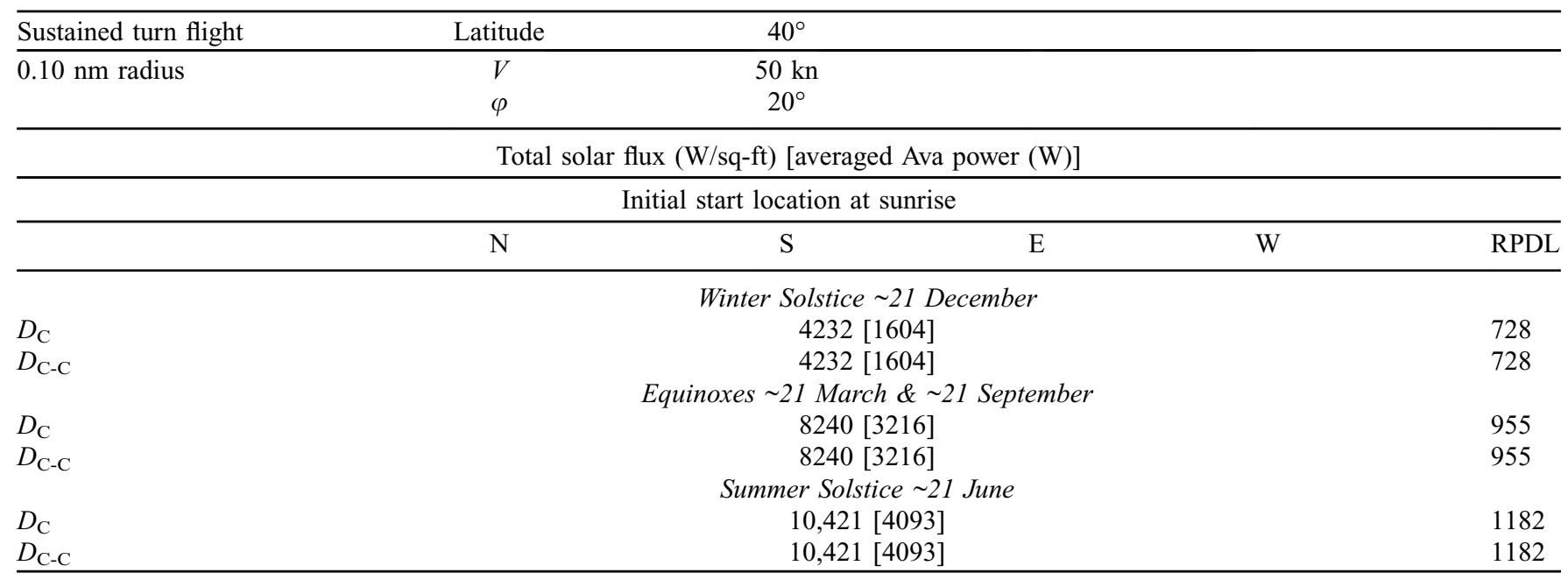

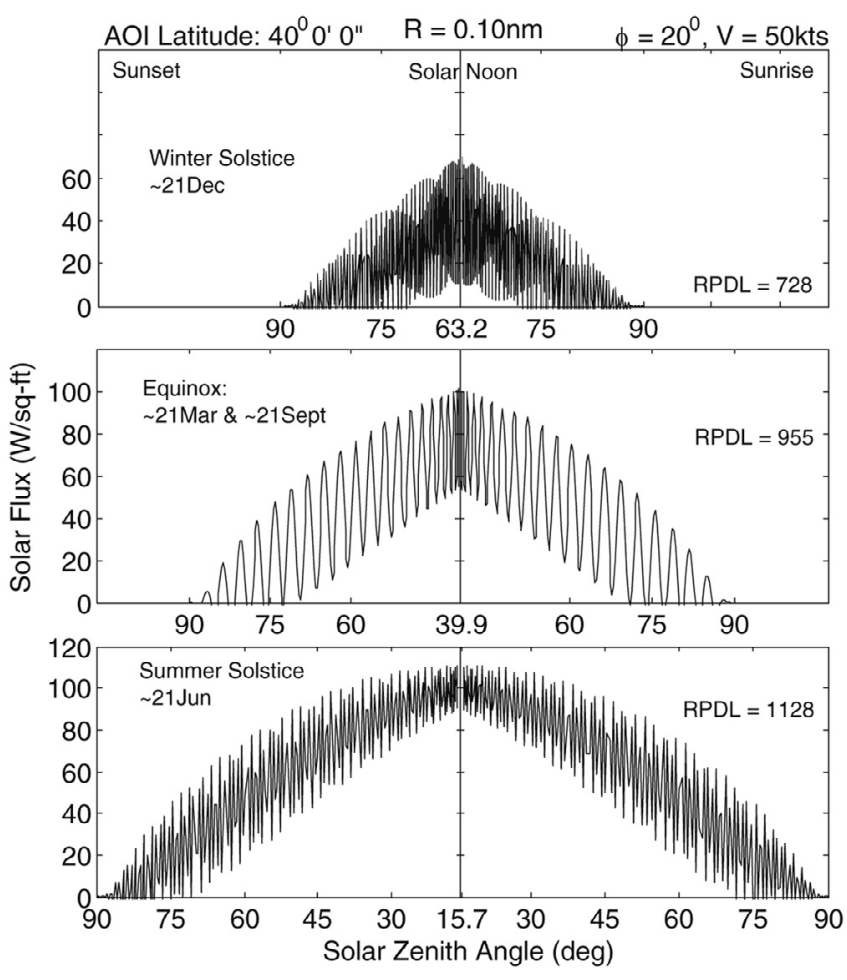

Figure 7. Sustained turn solar flux variations.

and latitude location in the inertial frame of reference, the solar flux was maximized for the corresponding vehicle bank angle and heading angle. The direct effect of solar flux was used, not accounting for the small contribution of diffusion; however, by maximizing the direct flux, the flux due to diffusion is inherently maxed, refer to Figure 3 . The heading angle was transformed into the inertial frame of reference, including the effects of vehicle angle-of-attack. The flight window and objectives can be seen in Figure 9.

Referring to Figure 5, and allowing for a 20\% safety factor in vehicle speed above stall, the velocity for minimum power

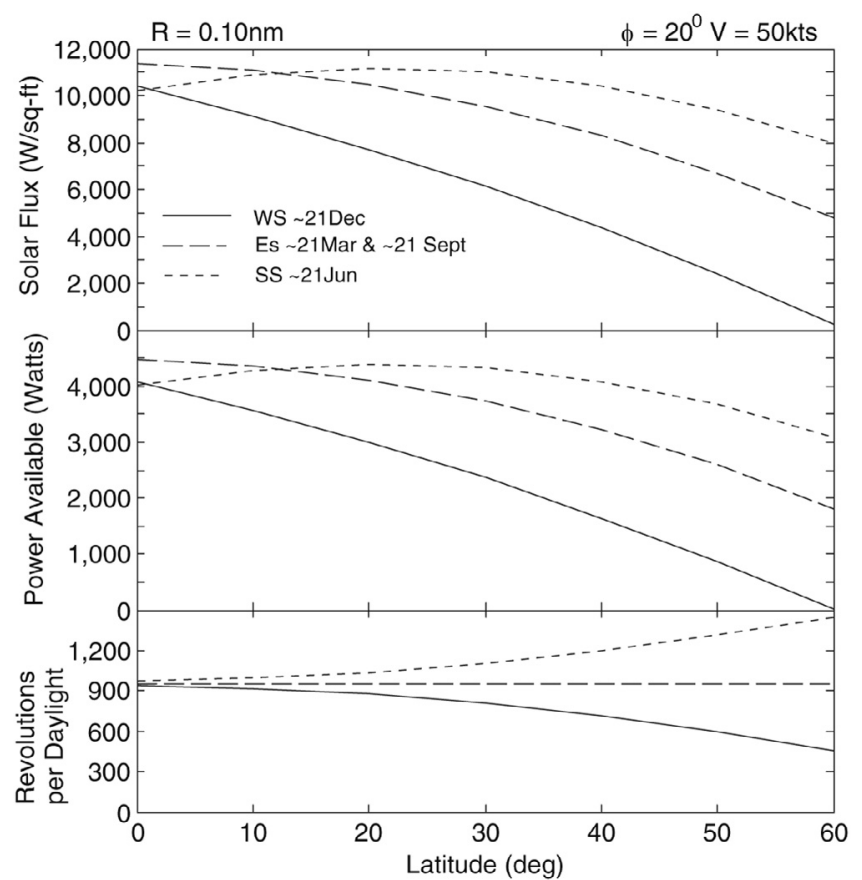

Figure 8. Total sustained turn solar flux and available power for latitude variations.

required for the UAV was set at a constant $50 \mathrm{kn}$, consistent with sustained turn loiter flight. The constant velocity flight satisfies the minimum power required, simplifying the pathplanning process and maximizing aerodynamic efficiency for the vehicle in level flight with a constant angle-of-attack of $10^{\circ}$. Considering the discussed modeling for the vehicle path-planning, Figure 10 shows the DOPP technique for the representative UAV for solar-regenerative high altitude long endurance flight.

The "driver" conditions are constrained by the bank angle constraint $\left(g_{1}\right)$; with the objective of maximizing solar flux (solar power) throughout the vehicle's flight window when 


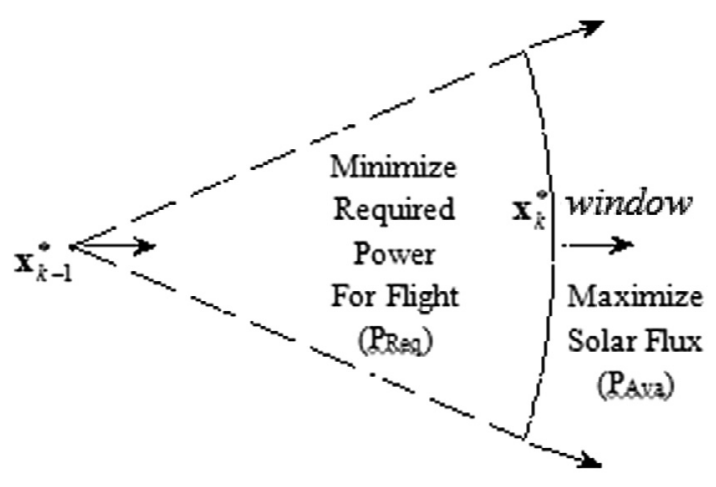

Figure 9. Flight maneuvering window and objectives.

$$
\begin{aligned}
& \text { Driver: } \\
& \text { Maximize } F^{(\text {direct })}=S_{0} \mu e^{-\tau / \mu_{0}} \text {; } \\
& S_{0} \equiv \text { Solar Cons } \tan t \\
& \tau \equiv \text { Optical Thickness } \\
& \mu_{o}=\cos Z \equiv \text { Solar Zenith } \\
& \mu=\cos \theta \equiv \text { Surface Obliquity } \\
& \cos \theta=\cos Z \cos \Delta+\sin Z \sin \Delta \cos (A-\psi) \\
& \text { Subject to: } g_{1}=\left|\phi_{m x}-\phi\right| \geq 0 \text {; } \\
& \begin{array}{l}
g_{2}=\left({\text { Longitude } \left.- \text { Longitude }_{A O I}\right)^{2}}_{\text {with b.c. }}-\left({\text { Latitude } \left.- \text { Latitude }_{A O I}\right)^{2} \leq R_{R-O-S}^{2}}^{\text {with solution }}\right.\right.
\end{array}
\end{aligned}
$$

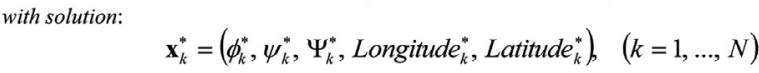

$$
\begin{aligned}
& \text { Path: } \\
& \text { Minimize } P_{\text {req }}(u)=J(u)=\int_{0}^{T} f_{0}(\mathbf{x}(t), u(t)) d t, \\
& \mathbf{x}(t),\left(\mathbf{x}_{k-1}^{*}(t), \ldots, \mathbf{x}_{k}^{*}(t)\right):[0, T] \rightarrow \Re^{n} \\
& \frac{d \mathbf{x}_{j}}{d t}=f_{j}(\mathbf{x}, u(t)), \quad(j=1, \ldots, n) \\
& \mathbf{x}(0)=\mathbf{x}_{k-1}^{*} ; \quad \mathbf{x}(T)=\mathbf{x}_{k}^{*} \\
& \text { where } \\
& J\left(u^{*}\right)=\min _{u \in U} J(u) \\
& \text { with } u^{*} \text { and the associated path } \mathbf{x} \text { called 'optimal.' }
\end{aligned}
$$

Figure 10. DOPP technique for solar-regenerative high altitude long endurance flight.

considering surface tilt $(\mu)$ at that specific time of day $\left(\mu_{0}\right)$. The second constraint $\left(g_{2}\right)$, boundary condition, assures that the vehicle stays within the defined range-of-sight window over the area of interest. The maximum solar power is determined along the "driver" window at each time step and sets the destination for the vehicle's path. The "path", in the case of the representative UAV is simplified by the constant velocity condition for minimizing power required for flight. The initial $\left(\mathbf{x}_{k-1}^{*}\right)$ and final (step, $\mathbf{x}_{k}^{*}$ ) destination for the vehicle is flown by a constant radius of curvature trajectory set by the ascertained bank angle $\left(\phi_{k}^{*}\right)$ for the vehicle's "driver" optimum destination.
The procedure for the on-line analysis requires a total of six updating adaptive processes from sunrise to sunset. The flow of processes can be seen in Figure 11.

The first requires the definition of area of interest (AOI), with equation of time, the solar and geocentric coordinates, and hours of daylight including sunrise/sunset location for the time-of-year of interest. The second, independent process is the definition of the available flight window based on the representative UAV kinematics and the user defined radius of flight window for the "driver" window objective analysis. The first "driver" objective constraint $\left(g_{1}\right)$ limits the maximum bank angle of the vehicle. Next, the flight window, area of interest, and time-of-year information is combined and transferred into the Earth inertial frame of reference. The second boundary condition constraint $\left(g_{2}\right)$ for the "driver" objective insures that the UAV stays within the available range-of-sight for continual surveillance over the area. Parameters for solar flux measurements are determined along "driver" window. Following, the available solar flux along with the vehicle flight window is determined. The UAV maximum solar flux "driver" objective is then determined using an adaptive- $\mathrm{A}^{*}$ search procedure in determining the necessary parameters $\mathbf{x}_{k}^{*}$ conditions to be met. That information is then used to determine flight path for minimum power required in order to meet the forward step destination and vehicle orientation. This process is iterated from initial location at sunrise through sunset.

This process is "locally" optimal for increasing the adaptability and on-line capability for multi-objective approach for solar powered flight. The DOPP technique only projects forward one step at a time until the overall goal is met. As an example of the local optimality and the pros and cons consider the UAV directly over the area of interest and initially heading north at sunrise. Figure 12 shows path of the vehicle for the first 50 min of flight.

The vehicle has a northerly initial heading for the example figure, during sunrise. In order to obtain a maximum amount of solar flux, the UAV banks a positive $20^{\circ}$, leaning the upper solar panel wing surface into the Sun's intensity. Even though the technique is maximizing solar flux at each step, because it does not project meta-paths, like an off-line analysis, it is limited to a single flight trajectory. The result with starting on a single path may require compromise to reach the end goal. For the flight duration shown above the vehicle starts a sustained turn flight banking the solar panel surface into the Sun, but as it projects further along the flight path there is a decrease in maximum solar flux. However, the DOPP technique adapts and quickly brings a change to the flight trajectory to further increase the maximum solar flux. This can be seen in Figure 13, for the maximum solar flux variation from the example flight of Figure 12.

The small amount of max obtained solar flux is due to the nature of the given example during sunrise when the Sun is close to the horizon and around a $90^{\circ}$ zenith angle.

Predicting further steps ahead to determine, by selecting an alternate (non-optimal) destination, there may be meta-paths in which overall produces more solar power. However, this would require a time intensive and limited off-line analysis, and would void the purpose of introducing real-time path-planning adaptability in furthering UAV-autonomy. 


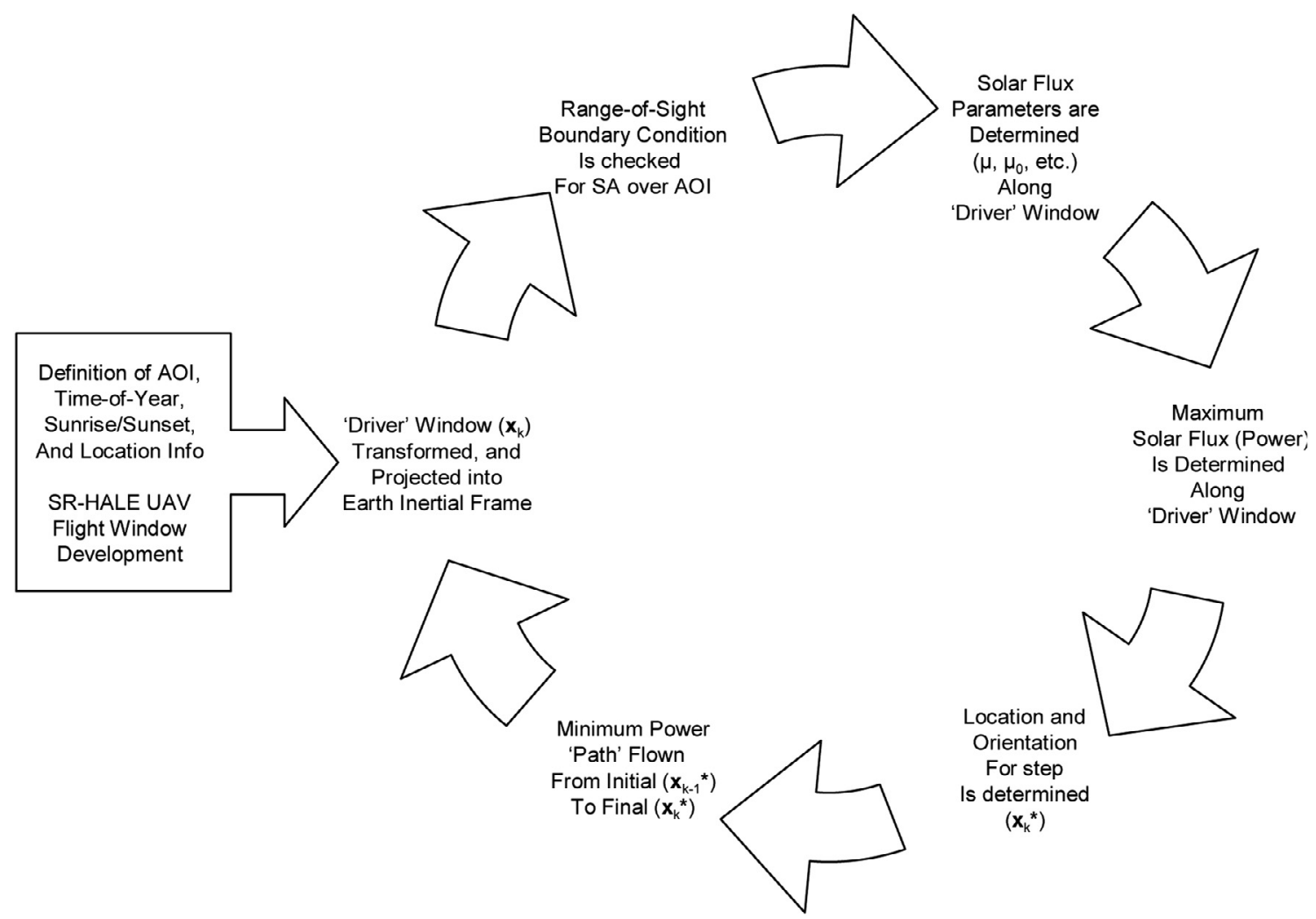

Figure 11. DOPP flow chart for solar-regenerative high altitude long endurance flight.

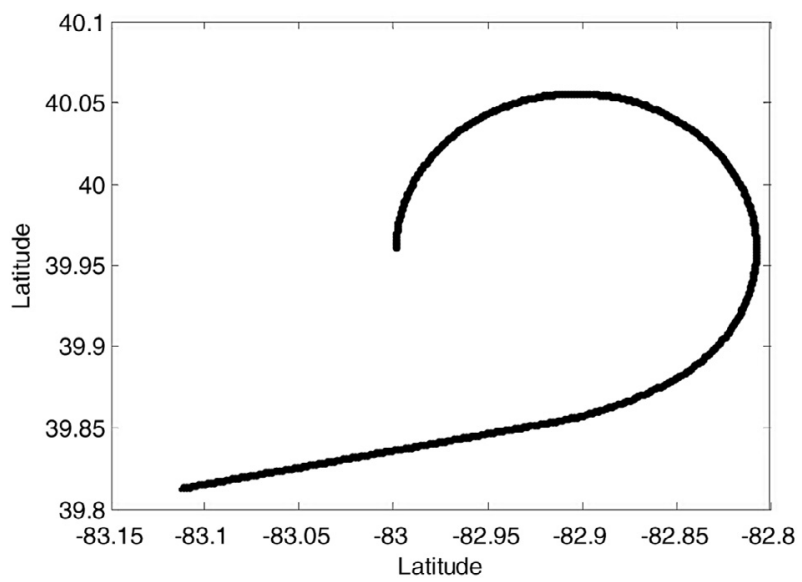

Figure 12. DOPP example flight at sunrise.

\section{Case-study results}

Consider the full day of flight on the summer solstice $(\sim 21$ June) over the area of interest at $40^{\circ}$ latitude, and with the initial location of the vehicle was set directly over the area and heading north. Figure 14 shows the flight pattern, with and without the range-of-sight boundary correction of the UAV for the entire day. The path outside of the range-of-sight circle is in violation of the second boundary condition constraint. Implementing the constraint requires the vehicle to bank hard right and maintains continual situational awareness over the area of interest.

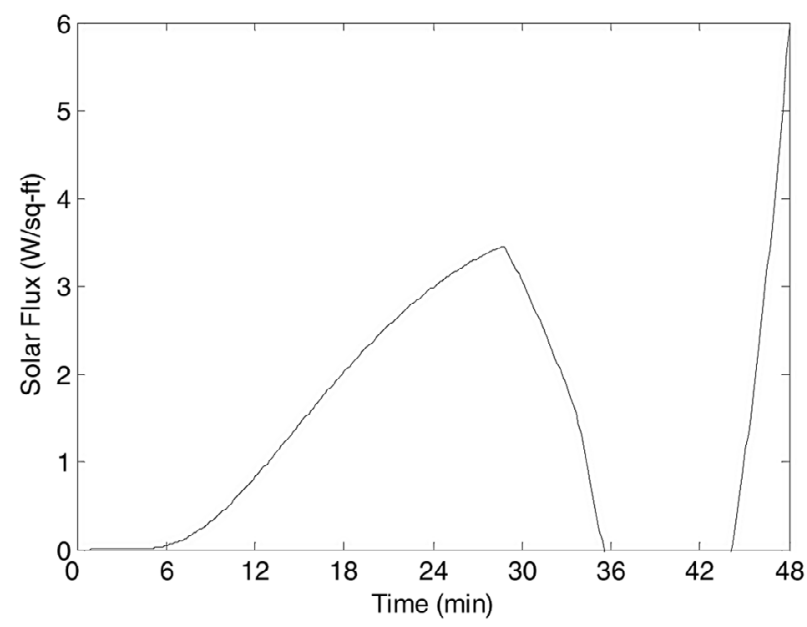

Figure 13. DOPP example of local optimality process: sunrise.

As discussed previously, the figure shows that during sunrise $\left(Z=90^{\circ}\right)$ the vehicle is in a sustained bank. As the solar zenith angle decreases the vehicle path requires less sustained banking flight to orient the solar panel surface into the Sun's rays for solar power. The total solar flux gather for the day was $12,838 \mathrm{~W} / \mathrm{ft}^{2}$ $\left(1193 \mathrm{~W} / \mathrm{m}^{2}\right)$, an averaged available power of $5100 \mathrm{~W}$. When compared to the baseline loiter, sustained turn flight at a $20^{\circ}$ bank angle, this resulted in a $19.7 \%$ increase in averaged available power; and a $2.0 \%$ decrease in power required for flight.

To obtain a better appreciation for the technique's capability, consider the solar flux variation from sunrise to sunset for 


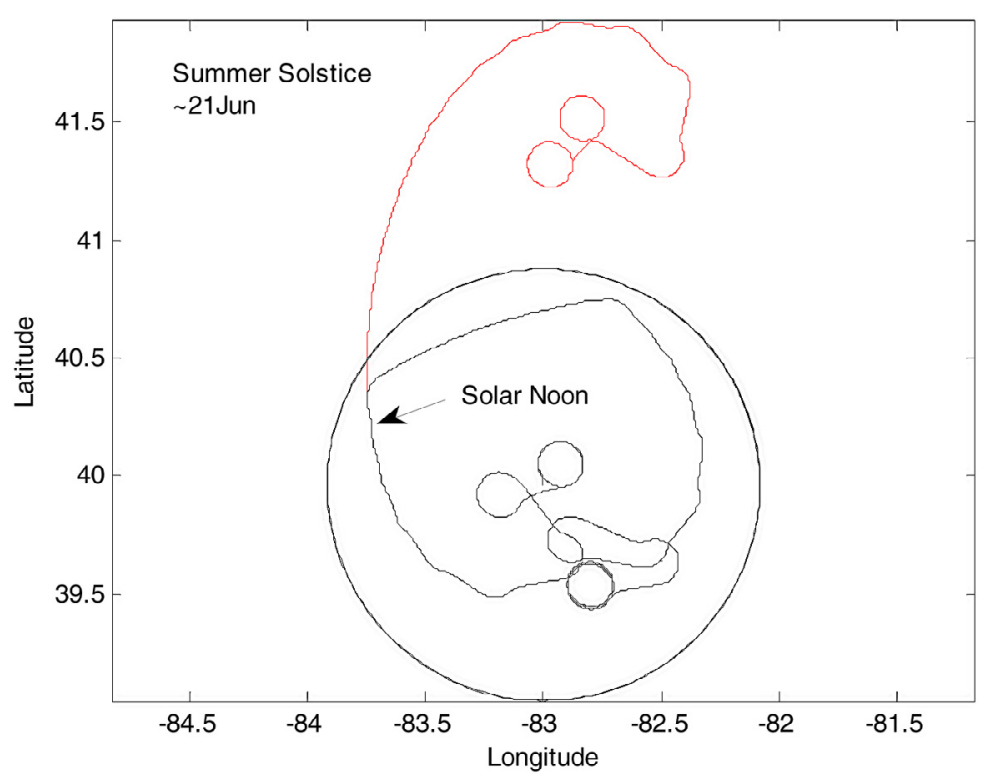

Figure 14. DOPP flight pattern on summer solstice.
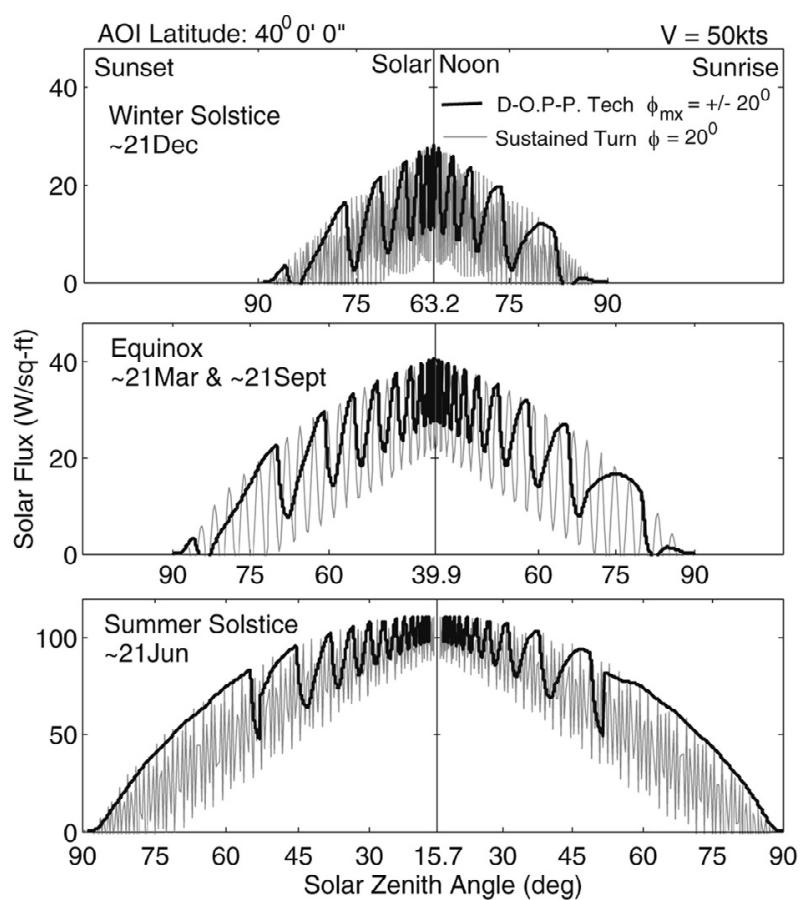

Figure 15. DOPP technique solar flux variation comparisons with the baseline.

the DOPP flight pattern compared to the baseline sustained turn (Figure 15). The winter and summer solstice and equinox(s) times-of-year were considered.

Figure 15 shows that as the Sun approaches solar noon, the vehicle's bank angle becomes more sensitive since the variation of solar flux across the "driver" window becomes less.

Before continuing the analysis, consider two conditions that have an effect on the optimal flight technique. First, consider the effects of distance in which the "driver" window is projected forward of the UAV. The results shown above were

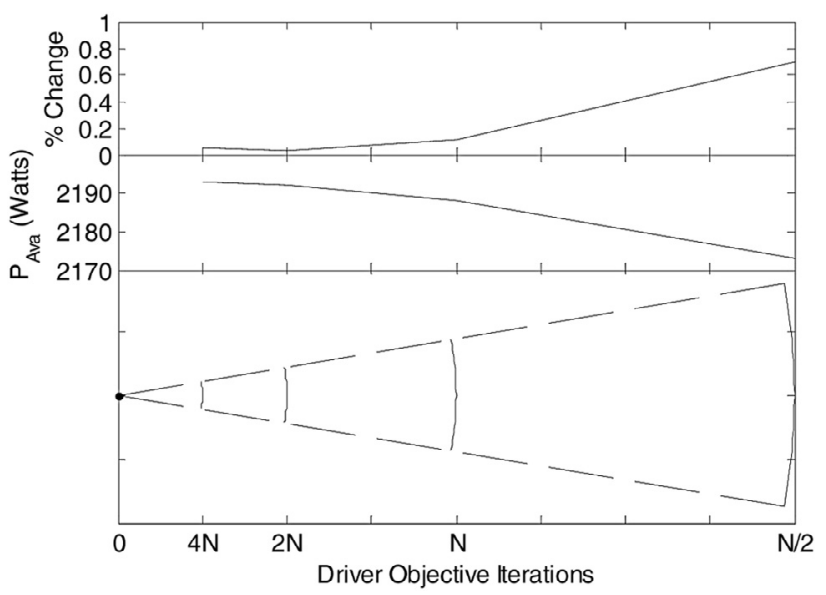

Figure 16. DOPP driver window projection effects on available power.

for a projection window distance of 0.01 nautical-miles with $N$ iterations. The effects on the maximum total available power and percent differences for varying the number of iterations is shown in Figure 16. The conditions shown in the figure are during the winter solstice, the worst case condition, showing the largest differences and the potential for most improvement.

The figure shows that with further increase in number of iterations, decreasing projection of "driver" window, that the improvements determined are within less than $0.1 \%$ difference. The adverse effects of adding computation time required for decreasing the project window outweighs the minimal gain in performance. Other considerations must be accounted for when increasing the projection distance, decreasing the number of iterations, other than the significant loss in improvement shown above. As the "driver" window is projected farther, the "path" for the vehicle does not become apparent; meaning, that the actual path for the vehicle to meet its destination may not be simply a sustained turn to meet its objective. For the 
Table 4. Effects of varying UAV bank angle limit.

\begin{tabular}{|c|c|c|c|c|}
\hline \multicolumn{5}{|c|}{ Case study location } \\
\hline Longitude: & $82^{\circ} 59^{\prime} 56^{\prime \prime}$ & West & $(-82.99889 \mathrm{~W})$ & $V=50 \mathrm{kn}$ \\
\hline Latitude: & $39^{\circ} 57^{\prime} 40^{\prime \prime}$ & North & $(39.96111 \mathrm{~N})$ & \\
\hline \multicolumn{2}{|c|}{ D-O.P-P. Technique } & \multicolumn{2}{|c|}{ Summer solstice $\sim 21$ June } & $\%$ Increase \\
\hline$\varphi_{\mathrm{mx}} \operatorname{limit}\left({ }^{\circ}\right)$ & $F_{\text {total }}(\mathrm{W} / \mathrm{sq}-\mathrm{ft})$ & Ave $P_{\text {Ava }}(\mathrm{W})$ & Ave $P_{\text {Req }}(\mathrm{W})$ & Ava/Req \\
\hline \pm 10 & 11,000 & 4325 & 3157 & \\
\hline \pm 20 & 12,838 & 5065 & 3259 & $14 \% / 3.1 \%$ \\
\hline \pm 30 & 14,349 & 5672 & 3530 & $10 \% / 8 \%$ \\
\hline
\end{tabular}

Table 5. Flight maneuvering comparisons.

\begin{tabular}{|c|c|c|c|c|}
\hline \multicolumn{5}{|c|}{ Case study location } \\
\hline Longitude & $82^{\circ} 59^{\prime} 56^{\prime \prime}$ West & $(-82.99889 \mathrm{~W})$ & Sustained turn $\varphi=20^{\circ}$ & $50 \mathrm{kn}$ \\
\hline Latitude & $39^{\circ} 57^{\prime} 40^{\prime \prime}$ North & $(39.96111 \mathrm{~N})$ & D-O.P-P. $\varphi=0^{\circ}- \pm 20^{\circ}$ & $50 \mathrm{kn}$ \\
\hline Maneuver & Averaged available & & Averaged power required & \\
\hline \multicolumn{5}{|c|}{ Winter Solstice 21 December } \\
\hline Sustained turn & $1604 \mathrm{~W}$ & & $3346 \mathrm{~W}$ & \\
\hline D-O.P-P. & $2214 \mathrm{~W}$ & $27.6 \%$ & $3268 \mathrm{~W}$ & $-2.4 \%$ \\
\hline \multicolumn{5}{|c|}{ Equinoxes $\sim 21$ March \& 21 September } \\
\hline Sustained turn & $3221 \mathrm{~W}$ & & $3346 \mathrm{~W}$ & \\
\hline D-O.P-P. & $3838 \mathrm{~W}$ & $16.1 \%$ & $3259 \mathrm{~W}$ & $-2.7 \%$ \\
\hline \multicolumn{5}{|c|}{ Summer Solstice $\sim 21$ June } \\
\hline Sustained turn & $4093 \mathrm{~W}$ & & $3346 \mathrm{~W}$ & \\
\hline D-O.P-P. & $5100 \mathrm{~W}$ & $19.7 \%$ & $3280 \mathrm{~W}$ & $-2.0 \%$ \\
\hline
\end{tabular}

remainder of the analysis, the projection window was maintained at its initial location.

Next, consider the effects of varying the bank angle limit for the UAV. Table 4 shows the effects on total available solar flux, and both averaged power available and required for the flight using the DOPP technique.

By increasing the bound from $\pm 10^{\circ}$ to $\pm 20^{\circ}$ there is a significant improvement (14\%) in averaged available solar power with a relatively small increase in required power $(3.1 \%)$. With further increase in bank angle limit, the tradeoff becomes less significant. If the vehicle structural integrity allowed for a bank angle limit of $\pm 30^{\circ}$, there would be a $10 \%$ improvement in available power, but subsequently an $8 \%$ increase in required power as well.

Continuing the analysis and comparisons, Table 5 are the summary results comparing the maximum obtainable solar power and the power required for sustained turn, and the average power required for the vehicle flying with the adaptive DOPP process. The summary results are during the equinoxes and summer and winter solstices over the case-study area of interest.

The DOPP technique increased the available solar power by $27 \%$ during the winter solstice, the time-of-year with minimum amount of daylight, while decreasing the power required for flight by $2 \%$ throughout the day. During the equinoxes and summer solstice similar improvements resulted, demonstrating the benefits of the path-planning technique and its application to solar regenerative high altitude long endurance surveillance flight.

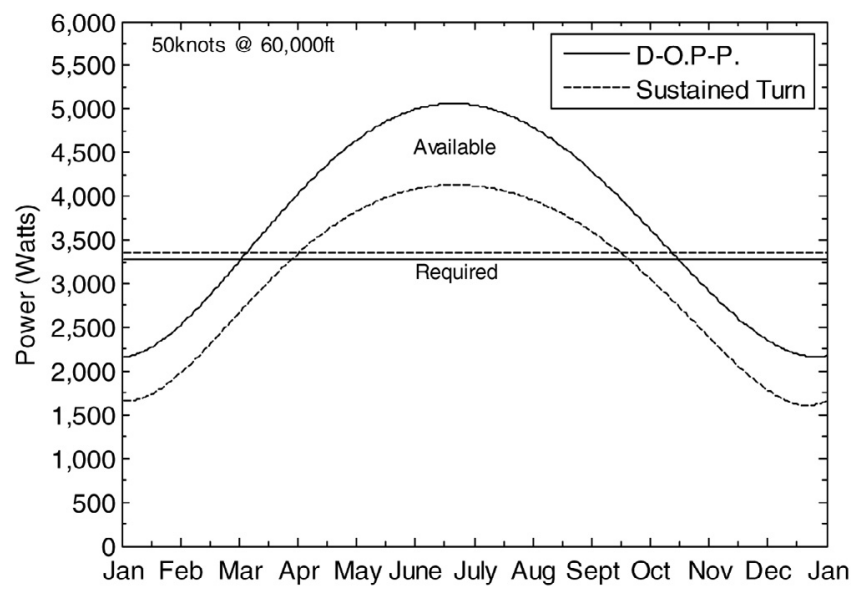

Figure 17. Averaged power comparison for a year at $40^{\circ}$ latitude.

The optimum flight patterns and the daily averaged solar flux and solar power gathered was analyzed and compared to the baseline sustained turn flight for an entire year over the area of interest (Figure 17).

For the UAV flying a sustained turn loiter over the area of interest, the available flight time throughout the year for the vehicle to obtain enough solar power to maintain flight would be approximately between 1 April and 13 September, 165 days total. Using the DOPP technique for generating the flight path, the available flight window over the area increased to total of 222 days, 4 March-12 October. This is an additional 57 days 


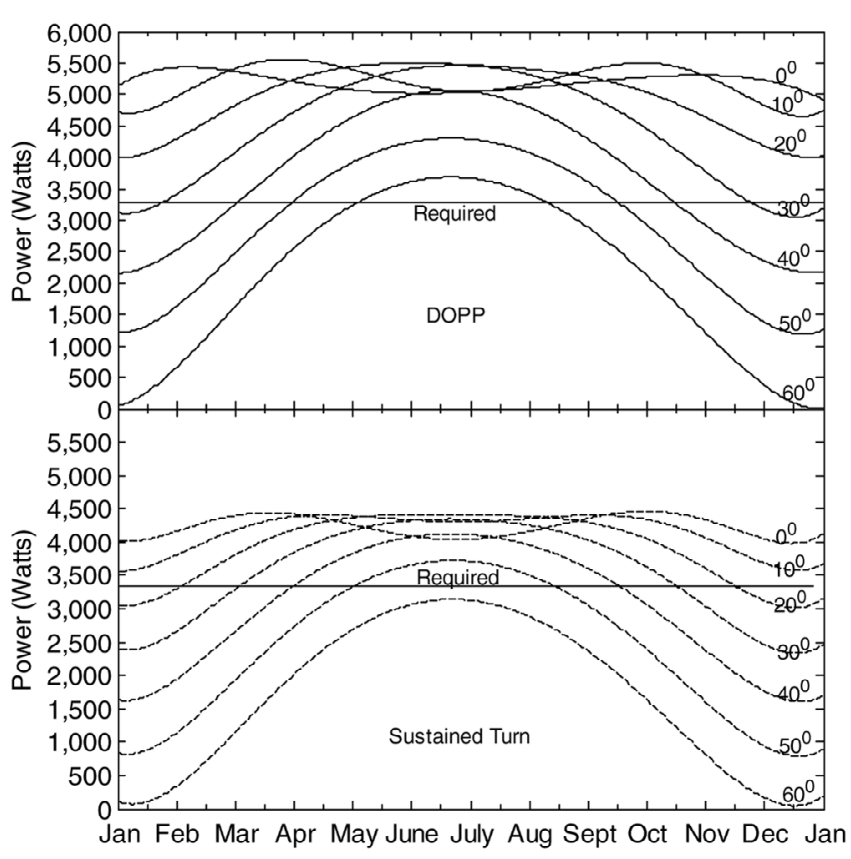

Figure 18. Latitude location effects on the averaged power variation.

of available flight for the year, a $26 \%$ improvement over the sustained turn loiter flight.

Similarly, the daily averaged available power for the optimum flight patterns was compared with the baseline sustained turn for variations in latitude from the equator to $60^{\circ}$ (Figure 18).

The analysis was limited to latitudes less than $60^{\circ}$. For latitudes greater than $60^{\circ}$, there is not enough sufficient averaged available power to meet the required power for the representative UAV to maintain flight.

From Figure 18, flying a sustained turn loiter pattern was limited to $50^{\circ}$ latitude or less. However when flying the DOPP pattern, the vehicle is capable of increasing the flight time at each latitude by approximately 2 months, including an approximate 2 months flight period at $60^{\circ}$ latitude.

\subsection{High altitude wind effects}

Accounting for high altitude winds $\left(V_{\mathrm{w}}\right)$ in the UAV's kinematic modeling equation (9), Figure 19 is the corresponding example of the first hour of flight. This was compared with the no wind example (Figure 12) during sunrise.

The effects of a wind velocity of $10 \mathrm{kn}$ at a W-S-W heading $\left(\Psi_{\mathrm{w}}=260^{\circ}\right.$ Inertial Earth frame $)$ are shown, typical conditions that would be seen during the spring and summer months. The vehicle has a northerly initial heading and the high altitude wind affected "driver" window is shown to project the flight window, "pushing" the vehicle further east. When considering the entire day, for this example, the total solar flux available decreased (compared to Table 4) by $1.8 \%$, and increased the averaged power required by less than $0.1 \%$.

A few important comments on wind effects: first, the high altitude wind effects and the resultant available solar flux and

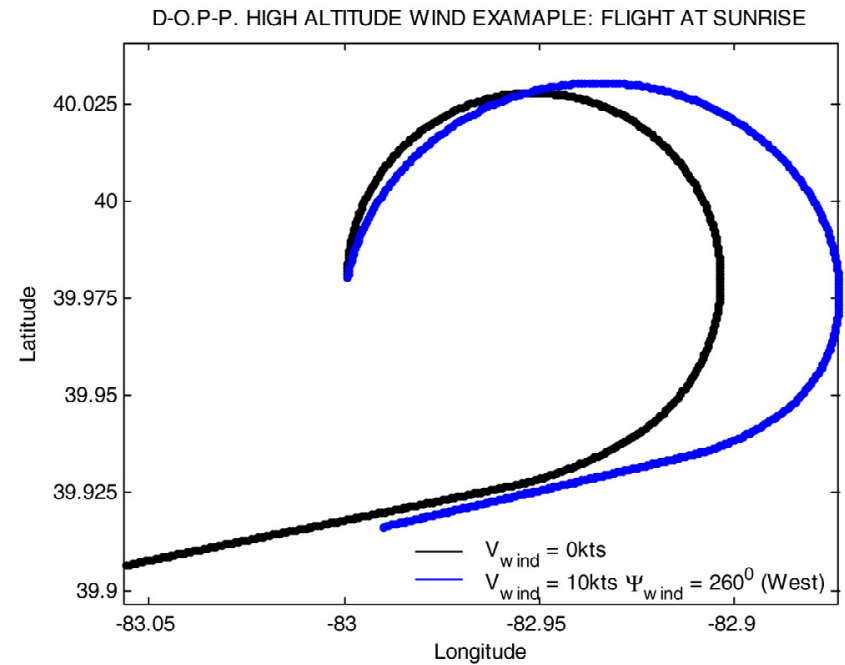

Figure 19. High altitude wind example flight at sunrise.

averaged power required for UAV flight were determined to be a case-by-case study. There were no observable trends for either the increase or decrease in available solar flux with variations in time-of-year or high altitude wind conditions, supporting the need for on-line adaptive capability for a high altitude solar powered flight path generation. Second, as the wind speed increased, an increase in vehicle speed was needed to compensate. For this condition, the increase in vehicle speed allowed for flexibility in vehicle movement for orientation increasing available solar power. However, this is not without the cost of increasing power required. Finally, and most significant, there was always a noticeable significant increase in available solar flux for flight with DOPP process over the baseline sustained turn flight, regardless of wind conditions.

\section{Conclusions}

The following introduced a multi-objective dual-optimal path-planning technique for unmanned air vehicle path and trajectory autonomy generation, through task allocation and sensor fusion. The dynamic optimization technique generates online adaptive flight paths for vehicle based on available flight windows and environmental influenced objectives. The realtime path generation for aircraft considered multiple independent conflicting objectives without the necessity of developing artificial parameters. The on-line process was locally-optimal based and adaptive, reducing the significant size in decision space required for off-line path search techniques. The technique was not limited to individual methods or path planning algorithms, however, certain procedures, like A* search proved advantageous in real-time analysis.

Solar powered high altitude long endurance flight was investigated as a case-study of the dual-optimal path-planning technique. The flight mechanics of a flight test proven representative aircraft was used, and a case-study example location in which to support continual situational awareness was chosen. The vehicle was limited to a constant altitude of $60,000 \mathrm{ft}(18.3 \mathrm{~km})$ and high altitude winds for the area of 
interest were considered. At that altitude the range-of-sight, i.e. the limited geographical flight window, was determined by existing surveillance equipment common onboard high altitude aircraft. The surveillance system and the determined flight constraint window assured continual surveillance capability over the area of interest. Solar panels, covering $80 \%$ of the upper surface of the UAV wing, electric motors, and energy storage system were selected based on existing flight test demonstrated equipment.

A common sustained turn loiter pattern was considered a baseline fixed flight maneuver for comparisons. Sustained turn analysis consisted of varying location and time-of-year as well as the vehicle's bank angle, flight velocity, turn radius, direction of flight, and initial starting location. The variation of each flight condition was individually considered to determine the trends, if any, and their effects for solar powered flight. It was shown, that the latitude location and time-of-year varied the available solar flux significantly. The other, vehicle dependent flight conditions were determined over the case-study area of interest at $40^{\circ}$ latitude. It was determined that variations in flight velocity and the turning radius trends were consistent, a result that was utilized not only in determining baseline conditions but in dual-optimal path-planning modeling and analysis as well.

The objectives of the optimization technique were to determine the minimum required power flight paths to the predetermined location and orientation for obtaining maximum solar flux established by the "driver". This was done for still wind and constant wind conditions at altitude over the area of interest and for variations in latitude. The aircraft kinematics was used to develop the flight maneuvering window and was constrained by a maximum bank angle of $\pm 20^{\circ}$. It was determined that minimum power required occurred at the vehicles stall speed. To account for this the flight velocity was set to a constant $50 \mathrm{kn}, 20 \%$ safety margin, while maintaining minimum power required. The projection of the flight window was set at one nautical-mile, assuring sufficient response time for the vehicle.

The path-planning technique increased the total available solar flux by as much as $20 \%$, and decreased the average power required for flight compared to the baseline sustained turn loiter flight for the case-study area of interest over the time span of one year. This increased the total flight time over the area throughout the year by an additional 57 days, a 26\% improvement over the baseline loiter flight. Considering latitude variations from the equator through $60^{\circ}$, the technique increased the available flight time consistently by an approximate 2 months, including flight at $60^{\circ}$ latitude where the baseline flight pattern was unattainable. Finally, when considering high altitude wind effects, the results showed that the physical flight path were altered; however, regardless if high altitude wind effects were considered the DOPP technique significantly improved the flight capability over the baseline conditions.

\section{Implication and influences}

The published work has an impact in providing continual development of real-time autonomous optimal path-planning and trajectory generation capability for unmanned vehicle systems. The technique developed and demonstrated is an adaptive path generation for vehicles considering multiple independent conflicting objectives without the necessity of developing artificial or weighting parameters. The technique can be applied to unmanned air, ground, and sea based vehicles. The technique is capable of adapting existing optimization algorithms into the dual-optimal path-planning model. Additional implications of the technique to real-time optimal path-planning for unmanned air systems that could have a significant impact include, but not limited to: cooperative tactics between unmanned air vehicles, dynamic target pursuit, and evading potential threats.

\section{References}

1. Trefz JL Jr. 2003. From persistent ISR to precision strikes: the expanding role of UAVS, LCDR, US Navy, Navy War College: Newport, RI.

2. Mittal S, Deb K. 2007. Three-dimensional offline path planning for UAVs using multiobjective evolutionary algorithms. Indian Institute of Technology: Kanpur, India.

3. McGee TG, Spry S, Hedrick K. 2005. Optimal path planning in a constant wind with a bounded turning rate. Center for Collaborative Control for Unmanned Vehicles, University of California: Berkeley, CA.

4. McGee TG, Hedrick K. 2007. Optimal path planning with a kinematic airplane model. Journal of Guidance, Control, and Navigation, 30(2), 629-633.

5. Bestaoui Y, Dahmani H, Belharet K. 2009. Geometry of translational trajectories for an autonomous aerospace vehicle with wind effect, in 47th AIAA Aerospace Sciences Meeting Including the New Horizons Forum and Aerospace Exposition, Orlando, FA.

6. Shapira I, Ben-Asher JZ. 1997. Near-optimal horizontal trajectories for autonomous air vehicles. Journal of Guidance, Control, and Navigation, 20(4), 735-741.

7. Erzberger H, Lee H. 1971. Optimum horizontal guidance techniques for aircraft. Journal of Aircraft, 8(2), 95-101.

8. Tsitsiklis JN. 1995. Efficient algorithms for globally optimal trajectories. IEEE Transactions on Automatic Control, 40(9), $1528-1538$.

9. McManus IA, Walker RA. 2006. Multidisciplinary approach to intelligent unmanned-airborne-vehicle mission planning. Journal of Aircraft, 43(2), 318-335.

10. Fahroo F, Ross IM. 2002. Direct trajectory optimization by a Chebyshev pseudospectral method. Journal of Guidance, Control, and Dynamics, 25(1), 3860-3864.

11. Dogan A. 2003. Probabilistic path planning for UAVs, in 2 nd AIAA "Unmanned Unlimited" Systems, Technologies, and Operations, San Diego, CA.

12. Lennon JA, Atkins EM. 2004. Optimal path-planning with behavior based cost definition, in AIAA 1st Intelligent Systems Technical Conference, Chicago, IL.

13. Amin JN, Boskovic JD, Mehra RK. 2006. A fast and efficient approach to path planning for unmanned vehicles, in AIAA Guidance, Navigation, and Control Conference and Exhibit, Keystone, CO.

14. Geiger BR, Horn JF, DeLullo AM, Long LN. 2006. Optimal path planning of UAVs using direct collocation with nonlinear programming, in AIAA Guidance, Navigation, and Control Conference and Exhibit, Keystone, CO. 
15. Geiger BR, Horn JF. 2009. Neural network based trajectory optimization for unmanned aerial vehicles, in 47th AIAA Aerospace Sciences Meeting Including the New Horizons Forum and Aerospace Exposition, Orlando, FA.

16. Narayan P, Campbell D, Walker R. 2008. Multi-objective UAS flight management in time constrained low altitude local environments, in 46th AIAA Aerospace Sciences Meeting and Exhibit, Reno, NV.

17. Yokoyama N, Ochi Y. 2008. Optimal path planning for skid-toturn unmanned aerial vehicle, in AIAA Guidance, Navigation, and Control Conference and Exhibit, Honolulu, Hawaii.

18. Rippel E, Bar-Gill A, Shimkin N. 2005. Fast graph-search algorithms for general aviation flight trajectory generation. Technion - Israel Institute of Technology: Israel.

19. Chang W, Hsiao F, Sheu D. 2006. Two-point flight path planning using a fast graph-search algorithm. Journal of Aerospace Computing, Information, and Communication, 3, 453-488.

20. Schwartzentruber L, Foo JL, Winer EH. 2008. Three-dimensional multi-objective UAV path planner using meta-paths for decision making and visualization, in 12th AAIA/ISSMO Multidisciplinary Analysis and Optimization Conference, Victoria, British Columbia, Canada.

21. Miele A, Lee YL, Wu GD. Optimal trajectories for an aerospace plane, part 1: formulation, results, and analysis, Aero-Astronautics Report No. 247, Rice University, 1990.

22. Chieng W-Y. 2007. The study of flight path planning for multiple target visitations, Dissertation. Aeronautics \& Astronautics, etd-0613107-115826.

23. Frazzoli E, Dahleh MA, Feron E. 2002. Real-time motion planning for agile autonomous vehicles. Journal of Guidance, Control, and Navigation, 25(1), 1-48.

24. Pongpunwattana A, Rysdyk R. 2004. Real-time planning for multiple autonomous vehicles in dynamic uncertain environments. University of Washington: Seattle, WA.

25. Richards N, Sharma N, Ward D. 2002. A hybrid A*/automaton approach to on-line path planning with obstacle avoidance, in AIAA 1st Intelligent Systems Technical Conference, Chicago IL.

26. Howlett JK, McLain TW, Goodrich MA. 2006. Learning realtime A* path planner for unmanned air vehicle target sensing. Journal of Aerospace Computing, Information, and Communication, 3, 1-22.

27. Rathbun D, Capozzi B. 2003. Evolutionary approaches to path planning through uncertain environments, in AIAA's 1st Technical Conference and Workshop on Unmanned Aerospace Vehicles, Portsmouth, VA.

28. Pettit RL, Homer ML. 2004. An autonomous threat evasion response algorithm for unmanned air vehicles during low altitude flight, in AIAA 1st Intelligent Systems Technical Conference, Chicago, IL.

29. Geyer MS, Johnson EN. 2006. 3D obstacle avoidance in adversarial environments for unmanned aerial vehicles, in AIAA Guidance, Navigation, and Control Conference and Exhibit, Keystone, CO.

30. Kabamba PT, Meerkov SM, Zeitz FH III. 2006. Optimal path planning for unmanned combat aerial vehicles to defeat radar tracking. Journal of Guidance, Control, and Navigation, 29(2), 279-288.

31. Park JB, Vorsmann P. 2007. Strategies for the implementation of a sense and avoid system for unmanned air vehicles, in 3rd US-European Competition and Workshop on Micro Air Vehicle
Systems \& European Micro Air Vehicles, Conference and Flight Competition, Toulouse, France.

32. Tooren J, Heni M, Knoll A, Beck J. 2007. Development of an autonomous avoidance algorithm for UAVs in general airspace, EADS Defense \& Security, Military Air Systems: Munich, Germany.

33. Jorris TR, Cobb RG. 2008. Multiple method 2-D trajectory optimization satisfying waypoints and No-Fly zone constraints. Journal of Guidance, Control, and Dynamics, 31(3), 543-553.

34. Zengin U, Dogan A. 2004. Dynamic target pursuit by UAVs in probabilistic threat exposure map, in AIAA 3rd Unmanned Unlimited Technical Conference, Workshop and Exhibit, Chicago IL.

35. Caveney DK, Hedrick JK. 2005. Path planning for targets in close proximity with a bounded turn-rate aircraft, in AIAA Guidance, Navigation, and Control Conference and Exhibit, San Francisco CA.

36. Maj. Burns BS, Blue PA, Capt. Zollars MD. 2007. Simulation of real-time trajectory generator for automated aerial refueling with a required time of arrival, in AIAA Modeling and Simulation Technologies Conference and Exhibit, Hilton Head SC.

37. Sattigeri RJ, Johnson E, Calise AJ, Ha J. 2007. Vision-based target tracking with adaptive target state estimator, in AIAA Guidance, Navigation and Control Conference and Exhibit, Hilton Head SC.

38. Cloutier JR, Lin CF, Yang C. 1993. Maneuvering target tracking via smoothing and filtering through measurement concatenation. Journal of Guidance, Control, and Dynamics, 16(2), 377-384.

39. Han JD, Campbell M. 2003. Artificial potential guided evolutionary path plan for target pursuit and obstacle avoidance, in AIAA Guidance, Navigation, and Control Conference and Exhibit, Austin TX.

40. Larson RA, Mears MJ, Maj. Blue PA. 2005. Path planning for unmanned air vehicles to goal states in operational environments, in AIAA Infotech@Aerospace, Arlington VA.

41. Ousingsawat J, Campbell ME. 2004. On-line estimation and path planning for multiple vehicles in an uncertain environment. International Journal of Robust and Nonlinear Control, 14(8), 741-756.

42. Harl N. 2008. Coordinated rendezvous of unmanned air vehicles to a formation: a sliding mode approach, in AIAA Guidance, Navigation and Control Conference and Exhibit, Honolulu, Hawaii.

43. Bollino KP, Lewis LR. 2008. Collision-free multi-UAV optimal path planning and cooperative control for tactical applications, in AIAA Guidance, Navigation and Control Conference and Exhibit, Honolulu, Hawaii.

44. Boissonnat JD, Cerezo A, Leblond J. 1994. Shortest paths of bounded curvature in the plane. Journal of Intelligent and Robotic Systems, 11, 5-20.

45. Mahmoudian N, Woolsey CA, Geisbert J. 2007. Steady turns and optimal paths for underwater gliders, in AIAA Guidance, Navigation and Control Conference and Exhibit, Hilton Head, SC.

46. Foo JL, Knutzon JS, Oliver JH, Winer EH. 2007. Threedimensional multi-objective path planning of unmanned aerial vehicles using particle swarm optimization, in AIAA/ASME/ ASCE/ASC Structures, Structural Dynamics, and Materials Conference, Honolulu, Hawaii.

47. Swartzentruber L, Foo JL, Winer EH. 2009. Three-dimensional multi-objective UAV path planner using terrain information, in 
AIAA/ASME/ASCE/ASC Structures, Structural Dynamics, and Materials Conference, Palm Springs, CA.

48. Whitfield CA. An adaptive dual-optimal path-planning technique for unmanned air vehicles with applications to solarregenerative high altitude long endurance flight, Dissertation, The Ohio State University, Columbus, OH, 2009.

49. Weider S. 1982. An introduction to solar energy for scientists and engineers. John Wiley \& Sons, New York.
50. Strganac TW. Wind study for high altitude platform design, NASA-RP-1044, N80-12661, 1979.

51. Pearson J, Gregorek GM, Whitfield CA. High altitude morphing aircraft (HAMAC). AFRL-VA-WP-TR-2007-XXX- Final Report to USAF for Phase I Small Business Initiative, 2007.

52. Alemayehu D, Eaton E, Faruque I. 2004. HALE UAV: aerovironment pathfinder: aerodynamic and stability analysis, case-study: planform optimization. http://www.aoe.vt.edu/ $\sim$ mason/Mason_f/pathfinder.pdf

Cite this article as: Whitfield CA: An adaptive dual-optimal path-planning technique for unmanned air vehicles. Int. J. Simul. Multisci. Des. Optim., 2016, 7, A2. 